\title{
Molecular approaches for the detection of Schistosoma mansoni: possible applications in the detection of snail infection, monitoring of transmission sites, and diagnosis of human infection
}

\author{
Frederico GC Abath $/{ }^{+} /++$, Ana Lisa do Vale Gomes, Fábio L Melo, \\ Constança S Barbosa*/++, Roberto P Werkhauser
}

\begin{abstract}
Departamentos de Imunologia e *Parasitologia, Centro de Pesquisas Aggeu Magalhães-Fiocruz, Av. Prof. Moraes Rego s/nº Cidade Universitária, 50670-420 Recife, PE, Brasil
\end{abstract}

The detection of specific DNA sequences by polymerase chain reaction (PCR) has proved extremely valuable for the analysis of genetic disorders and the diagnosis of a variety of infectious disease pathogens. However, the application to the detection of Schistosoma mansoni is rare, despite a recommendation of the World Health Organization that a major focus of research on schistosomiasis should be on the development and evaluation of new strategies and tools for control of the disease. In this context, a few studies were published for the detection of the parasite in snails, monitoring of cercariae in water bodies, and diagnosis of human infection. The present minireview describes sensitive and specific PCR based systems to detect $\mathrm{S}$. mansoni, indicating possible applications in the detection of snail infection, monitoring of transmission sites, and diagnosis of human infection.

Key words: polymerase chain reaction - molecular diagnosis - schistosomiasis - transmission - snail - Schistosoma

Schistosomiasis is a disease transmitted by fresh water snails. It affects more than 200 million people worldwide and is endemic in 74 countries, with more than $80 \%$ of infected persons living in Africa. In Brazil, Schistosoma mansoni is the only causative species of schistosomiasis, and there are three species of intermediate hosts: Biomphalaria glabrata, B. straminea, and B. tenagophila. Adult $S$. mansoni live in the bloodstream. Their eggs pass out of the host with the faeces. In contact with water, free-swimming miracidia emerge from the egg and penetrate the soft tissues of the snail host. Within the snails, miracidia immediately differentiate into sporocysts and, as they migrate through the snail tissues, give rise to cercariae. Cercariae are shed from the snails and are infective to humans by direct penetration of the skin. Thus, molecular approaches can be used to detect DNA of different life cycle stages of $S$. mansoni by analyzing different biological samples: feces, snail tissues, and infested water bodies, resulting in diagnosis of vertebrate and invertebrate host infections, and identification of transmission sites.

The detection of specific DNA sequences by polymerase chain reaction (PCR) has proved extremely valuable for the analysis of genetic disorders and the diagnosis of a variety of infectious disease pathogens (Leal et al.

Financial support: Fiocruz internal funds (PDTIS), Facepe, $\mathrm{CNPq}$

${ }^{+}$Corresponding author: fabath@cpqam.fiocruz.br

${ }^{++} \mathrm{CNPq}$ research scholarships

Received 25 May 2006

Accepted 26 June 2006
1996, Lucena et al. 1998, Schindler et al. 2001, Rodrigues et al. 2002). However, the application to the detection of $S$. mansoni is rare, despite a recommendation of the World Health Organization that a major focus of research on schistosomiasis should be on the development and evaluation of new strategies and tools for control of the disease (WHO 2004). In this context, a few studies were published for the detection of the parasite in snails (Hanelt et al. 1997, Jannotti-Passos et al. 1997, Hamburger et al. 1998a, Melo et al. 2006), monitoring of cercariae in water bodies (Hamburger et al. 1998b), and diagnosis of human infection (Pontes et al. 2002). The use of PCR for diagnosis of human infection, using fecal samples was evaluated in patients living in endemic areas (Pontes et al. 2002). However, the approaches with potential for use in the identification of transmission sites (Hanelt et al. 1997, JannottiPassos et al. 1997, Hamburger et al. 1998a), were neither appropriate for large scale use nor formally validated in endemic areas. The sensitive and accurate identification of human infection, and the precise monitoring of schistosome transmission sites may be important tools for the long term control of the disease. The present minireview describes the development of sensitive and specific PCR based systems to detect $S$. mansoni DNA, indicating the possible applications of these techniques.

\section{Detection of $S$. mansoni by PCR}

Identification of snail infection - The identification of schistosome transmission sites involves the detection of S. mansoni infected snails by exposure of individual snails to artificial light and observation of cercarial shedding. Alternatively, snails may be crushed between glass slides and inspected for the presence of sporocysts. However, there are limitations to detecting the parasite in situations such as, low parasite burden, prepatent infections (detec- 
tion of cercarial shedding is only possible 30 days after infection), aborted development of sporocysts, and death of the molluscs after collection and prior to exposure to light (Barbosa 1992, Hanelt et al. 1997), resulting in understimation of the true prevalence of infection. The conventional techniques for identification of infected snails are simple, cheap, but time consuming and associated with high operational costs because of the necessity for trained personnel and an appropriate laboratory structure; the whole process involves collection of snails in suspected areas, as well as the maintenance and analysis of individual snails. In addition, discrimination of $S$. mansoni cercariae from other trematodes can be problematic. In the particular case of $B$. straminea, even the examination of many snails from endemic areas can be negative (Barbosa \& Silva 1992, Favre et al. 1995). Thus, conventional methods for the identification of transmission sites have limitations.

A nested PCR targeting the 18S rDNA of $S$. mansoni was developed for the identification of infection in snails (Hanelt et al. 1997). In another study, a PCR assay based on a highly repeated tandemly arranged DNA sequence was developed for detection of prepatent S. mansoni infection (Hamburger et al. 1998a). The use of PCR amplification of the minisatellite repeat from $S$. mansoni mitochondrial DNA was proposed to identify infected snails (Jannotti-Passos et al. 1997). As the latter two approaches result in a ladder profile reflecting the amplification of tandem repeats (Jannotti-Passos et al. 1997, Hamburger et al. 1998a), the possibility exists for some variation of the profile due to genetic variability, posing difficulties for the use of this procedure on large scale.

It is crucial that the assays discriminate $S$. mansoni from other parasites that may co-exist in naturally infected snails and which may pose problems for the differential diagnosis based on morphology (Chingwena et al. 2002, Hertel et al. 2003, Thiengo et al. 2004). We extended previous studies, by aligning multiple SSU rRNA sequences from vertebrate and invertebrate host, and trematodes closely related to $S$. mansoni, allowing for the design of very discriminative primers (Melo et al. 2006). Indeed, the novel PCR systems developed were highly specific for the detection of Schistosoma DNA, not amplifying, as suggested by multiple sequence alignment, DNA from vertebrate and invertebrate hosts of the parasite, or species representative of families of parasites commonly infecting Biomphalaria in Brazil (Thiengo et al. 2004).

Very recently, a PCR system targeting a specific $S$. haematobium tandemly repeated DNA sequence, termed Dra I (Hamburger et al. 2004), was used to study the dynamics of snail infection in endemic areas for schistosomiasis haematobium (Hamburger et al. 2004). A ladder pattern of amplicons was produced, and the detection limits was less than $10 \mathrm{fg}$. Nonetheless, it was based on DNA purification of individual snails, and the system was not considered practical for field use by these authors. Purification of DNA from pools of snails, followed by PCR is more appropriate for large scale screening in endemic areas. Thus, purification methods were adapted for processing pools of 25-50 B. glabrata (Melo et al. 2006). This decreases costs and allows for the use of molecular de- tection tools on a large scale. To evaluate the ability of the molecular approaches in detecting snail infection under natural conditions, snail pools (including B. glabrata and $B$. straminea) were collected in water bodies suspected of being transmission sites of schistosomiasis in the state of Pernambuco, Northeastern Brazil. The PCR based methods were significantly more efficient in detecting $S$. mansoni infection than the conventional method of induction of cercaria shedding (Melo et al. 2006).

The published PCR systems aimed at detecting $S$. mansoni DNA presented detection limits ranging from 1 fg to $1 \mathrm{pg}$, and were able to identify prepatent snail infections (Hanelt et al. 1997, Jannotti-Passos et al. 1997, Hamburger et al. 1998a). Detection of prepatency would capture the summation of factors affecting water contamination by schistosome eggs, and infectivity of miracidia (Hamburger et al. 2004). It has been shown that extraction of $10 \mathrm{~S}$. mansoni miracidia yields approximately $0.45 \mathrm{ng}$ DNA (Jannotti-Passos et al. 1997). Thus, it is estimated that 1 miracidium contains $\sim 45 \mathrm{pg}$ of genomic DNA. Since the genome of $S$. mansoni contains $\sim 580 \mathrm{fg}$, theoretically the PCR systems available can detect DNA corresponding to less than 1 miracidium, and the nested PCR variants can detect DNA corresponding to less than a single cell of the multicelular parasite $S$. mansoni.

Identification of infested water bodies - The risk of infection in transmission sites may be estimated by detecting infected snail capable of shedding cercariae. However, since seasonal fluctuations exist in snail population densities, infection rates, and cercarial output, information on both snail infection and presence and distribution of cercariae is required for evaluating the risk of infection (Hamburger et al. 1998b).

Schistosome cercariae are difficult to recover for identification in natural water bodies. The use of sentinel mice for this purpose is inaccurate, time consuming, and limited. Alternatively, methods for collection and morphogical identification are not appropriate for large scale use. Species identification of schistosome cercariae cannot be done by morphology, and since in many endemic areas animal schistosomes may appear together with human schistosomes in the same trasmission sites, species identification of schistosome cercariae is often required. To overcome the limitations of current methods for identification of cercariae in water, Hamburger et al. (1998b) developed a PCR for identifying $S$. mansoni cercariae. The relevant primers were designed based on a highly 121-bp repeated tandemly arranged DNA sequence that constitutes more than $10 \%$ of the genome of $S$. mansoni (Hamburger et al. 1991). Amplification of this sequence yields a ladder of products; the smallest band is $42 \mathrm{bp}$, and the others are progressively larger by 121-bp increments. The PCR assay should enable identification of cercariae even when the parasites are masked, or disintegrated. However, if the cercarial DNA persists for a long time in natural transmission sites, it may be detectable even after snails shedding cercariae are no longer present. The technique was able to detect $10 \mathrm{fg}$ of $S$. mansoni DNA, and consequently, a single cercaria suspended in water or trapped on a filter (Hamburger et al. 1998b). Thus, this approach may be 
useful for the definition of the distribution of cercariae in a site. Although the assay seems to be specfic, testing additional non-schistosome trematodes is still required for establishing the species-specificity (Hertel et al. 2004).

Diagnosis of human infection - The Kato-Katz parasitologic technique is currently the recommended method for diagnosing schistomiaisis mansoni because it is quantitiative, relatively inexpensive, and simple (Katz et al. 1972). However, the sensitivity of this technique decreases when the prevalence and intensity of infection are low (Ebrahim et al. 1997). Thus, this method is less efficient in areas of low endemicity, in post-treatment situations, and in the control of transmission. The usefulness of PCR for detecting $S$. mansoni DNA in human fecal samples was described (Pontes et al. 2002). These authors designed primers to target a tandem repeat DNA sequence of $S$. mansoni described previously (Hamburger et al. 1991). The specificity of this test was demonstrated by the absence of amplification when DNA from other helminthes (Ascaris lumbricoides, Ancylostoma duodenale, Taenia sollium, and Thrichiuris trichiuria), commonly infecting people in endemic areas for schistosomiasis were tested. Using an artificially prepared positive fecal sample, PCR was able to detect S. mansoni DNA in fecal samples containing 2 eggs/g, an amount not detectable by the Kato-Katz examination (detection limit $=20$ eggs/g). Subsequently, the same group reported the results of a more extensive comparison between the PCR assay and the parasitologic Kato-Katz technique, using fecal samples collected from individuals living in a Brazilian endemic area (Pontes et al. 2003). The prevalence of $S$. mansoni infection, calculated with one, two, or three KatoKatz examinations were $25.3,29.4$, and $30.9 \%$, respectively. The prevalence observed using the PCR technique was $38.1,13 \%$ higher than that determined with one Kato-Katz examination (Pontes et al. 2003). Taken collectively these data (Pontes et al. 2002, 2003) indicate that PCR detection of $S$. mansoni eggs in fecal samples is more sensitive than the conventional parasitological approach. Probably the molecular approach will not replace the conventional parasitological examination, due to the need of more sophisticated laboratory equipments and well trained personnel, and, consequently, operational costs. In addition, conventional PCR does not allow for quantification of parasite burden. Nonetheless, the PCR technique might prove to be especially useful in circumstances of lower intensity or prevalence of infection (Pontes et al. 2003), a condition for which the parasitologic examination shows a well-documented limitation of its sensitivity (Ebrahim et al. 1997).

The possibility of detecting $S$. mansoni DNA in sera of schistosomiasis patients was suggested (Pontes et al. 2002). However, the number of reported positive samples were very small, and it was not clear how many serum samples had been tested. Thus, it is still not demonstrated that there is enough free S. mansoni DNA in these kind of clinical samples to make them useful for PCR detection. We are currently investigating this issue by quantitative real time PCR (unpublished results). One of the advantages of real time PCR over conventional PCR is the pos- sibility to quantify parasite DNA in the samples, an indirect measure of the parasite burden.

\section{Conclusions}

In conclusion, the PCR systems available are sensitive, and have the potential to detect parasite DNA in snails, monitoring of cercariae in water bodies, and diagnosis of human infection. However, more extensive validation studies in endemic areas are crucial to fully investigate the suitability of the tools for these purposes, with benefits for the control of schistosomiasis. Quantitative real time PCR for S. mansoni detection, would have the additional advantage of estimating the parasite burden of the infection. Although the cost per reaction is still relatively high, the reagents and equipments for molecular techniques are becoming cheaper, making the discussion concerning high cost less relevant.

\section{REFERENCES}

Barbosa CS 1992. Methods for malacological work in schistosomiasis. Mem Inst Oswaldo Cruz 87 (Suppl. IV): 311312.

Barbosa CS, Silva CB 1992. Epidemiologia da esquistossomose mansonica no Engenho Bela Rosa, Município de São Lourenço da Mata, Pernambuco, Brazil. Cad Saúde Públ 8: 83-87.

Chingwena G, Mukaratirwa S, Kristensen TK, Chimbari M 2002. Susceptibility of freshwater snails to the amphistome Calicophoron microbothrium and the influence of the species on susceptibility of Bulinus tropicus to Schistosoma haematobium and Schistosoma mattheei infections. $J$ Parasitol 88: 880-883.

Ebrahim A, El-Morshedy H, Omer E, El-Daly S, Barakat R 1997. Evaluation of the Kato-Katz thick smear and formol ether sedimentation techniques for quantitative diagnosis of Schistosoma mansoni infection. Am J Trop Med Hyg 57: 706-708.

Favre TC, Bogea THP, Rotenberg L, Silva HS, Pieri OS 1995. Cercarial emergence of Schistosoma mansoni from Biomphalaria glabrata and Biomplahalaria straminea. Mem Inst Oswaldo Cruz 90: 565-567.

Hamburger J, He N, Xin XY, Ramzy RM, Jourdane J, Ruppel A 1998a. A polymerase chain reaction assay for detecting snails infected with bilharzia parasites (Schistosoma mansoni) from very early prepatency. Am J Trop Med Hyg 59: 872-876.

Hamburger J, Hoffman O, Kariuki HC, Muchiri EM, Ouma JH, Koech KK, Sturrock RF, King CH 2004. Large-scale polymerase chain reaction-based surveillance of Schistosoma haematobium DNA in snails from transmission sites in costal Kenya: a new tool for studying the dynamics of snail infection. Am J Trop Med Hyg 71: 765-773.

Hamburger J, Turetske T, Kapeller I, Deresiewicz R 1991. Highly repeated short DNA sequences in the genome of Schistosoma mansoni recognized by a species-specific probe. Mol Biochem Parasitol 44: 73-80.

Hamburger J, Yu-Xin X, Ramzy RM, Jourdane J, Ruppel A 1998b. Development and laboratory evaluation of a polymerase chain reaction for monitoring Schistosoma mansoni infestation of water. Am J Trop Med Hyg 59: 468-473. 
Hanelt B, Adema CM, Mansour MH, Loker ES 1997. Detection of Schistosoma mansoni in Biomphalaria using nestd PCR. J Parasitol 83: 387-394.

Hertel J, Haberl B, Hamburguer JHW 2003. Description of a tandem repeated DNA sequence of Echinostoma caproni and methods for its detection in snail and plankton samples. Parasitology 126: 443-449.

Hertel J, Kedves K, Hassan AHM, Haberl B, Haas W 2004. Detection of Schistosoma mansoni cercariae in plankton samples by PCR. Acta Trop 91: 43-46.

Jannotti-Passos LK, Vidigal THDA, Dias-Neto E, Pena SDJ, Simpson AJG, Dutra WO, Souza CP, Carvalho-Parra JF 1997. PCR amplification of the mitocondrial DNA minisatellite region to detect Schistosoma mansoni infection in Biomphalaria glabrata snails. Parasitology 83: 395-399.

Katz N, Chaves A, Pelegrino J 1972. A simple device for quantitative stool thick-smear technique in schistosomiasis mansoni. Rev Inst Med Trop São Paulo 14: 397-400.

Leal NC, Abath FGC, Alves LC, de Almeida AM 1996. A simple PCR-based procedure for plague diagnosis. Rev Inst Med Trop São Paulo 38: 371-373.

Lucena WA, Dhalia R, Abath FGC, Nicolas L, Regis LN, Furtado AF 1998. Diagnosis of Wuchereria bancrofti infection by the polymerase chain reaction using urine and day blood samples from amicrofilaraemic patients. Trans $R$ Soc Trop Med Hyg 92: 290-293.

Melo FL, Gomes ALG, Barbosa CS, Werkhauser RP, Abath FGC 2006. Development of molecular approaches for the identification of transmission sites of schistosomiasis. Trans $R$ Soc Trop Med Hyg (in press).

Pontes LA, Dias-Neto E, Rabello ALT 2002. Detection by polymerase chain reaction of Schistosoma mansoni DNA in human serum and feces. Am J Trop Med Hyg 66: 157162.

Pontes LA, Oliveira MC, Katz N, Dias-Neto E, Rabello A 2003. Comparison of a polymerase chain reaction and the katokatz technique for diagnosis infection with Schistosoma mansoni. Am J Trop Med Hyg 68: 652-656.

Rodrigues EHG, Brito MEF, Mendonça MG, Werkhäuser RP, Coutinho EM, Souza WV, Albuquerque MFPM, Jardim ML, Abath FGC 2002. Evaluation of PCR for Diagnosis of American Cutaneous Leishmaniasis in an Area of Endemicity in Northeastern Brazil. J Clin Microbiol 40: 3572-3576.

Schindler HC, Montenegro L, Montenegro R, Carvalho AB, Abath FGC, Jaureguiberry G 2001. Development and optimization of polymerase chain reaction-based malaria diagnostic methods and their comparison with quantitative buffy coat assay. Am J Trop Med Hyg 65: 355-361.

Thiengo SC, Mattos AC, Boaventura FM, Loureiro MS, Santos SB, Fernandez MA 2004. Freshwater snails and schistosomiasis mansoni in the state of Rio de Janeiro, Brazil: V Norte Fluminense Mesoregion. Mem Inst Oswaldo Cruz 99: 99-103.

WHO 2004. World Health Organization Special Programme for Research and Training in Tropical Disease. TDR home page at http://www.who.int/tdr/. 